\title{
Supplement to Technical Note: Conversion of Isoprene Hydroxy Hydroperoxides (ISOPOOH) on Metal Environmental Simulation Chamber Walls
}

Anne-Kathrin Bernhammer ${ }^{1,2}$, Martin Breitenlechner ${ }^{1, *}$, Frank N. Keutsch ${ }^{3}$, Armin Hansel ${ }^{1,2}$ and the 5 CLOUD Team ${ }^{4}$

${ }^{1}$ Institute for Ion and Applied Physics, University of Innsbruck, 6020 Innsbruck, Austria

${ }^{2}$ IONICON Analytik GmbH, 6020 Innsbruck, Austria

${ }^{3}$ John A. Paulson School of Engineering and Applied Sciences and Department of Chemistry and Chemical Biology, Harvard University, Cambridge, MA, USA

$10{ }^{4}$ CERN, Geneva, Switzerland

*now at: John A. Paulson School of Engineering and Applied Sciences and Department of Chemistry and Chemical Biology,

Harvard University, Cambridge, MA, USA

Correspondence to: Armin Hansel (스‥Hansel@uibk.ac.at)

Table S1: Structures and names according to the MCM Leeds as used in this paper

MCM Nomenclature

